\title{
THE INTELLIGENT GARBAGE DETECTION AND ALERT SYSTEM
}

\begin{tabular}{|c|c|c|c|}
\hline \multicolumn{4}{|c|}{$\begin{array}{c}\text { Dr Sarbananda Sahoo } \\
\text { SOMC, Sanskriti University, Mathura, Uttar Pradesh, India }\end{array}$} \\
\hline Journal & \multicolumn{3}{|c|}{$\begin{array}{l}\text { Samvakti Journal of Research in Information Technology } \\
\text { https://www.sjrit.samvaktijournals.com } \\
\text { Volume } 2 \text { Year of Volume } 2021 \text { Page No : } 74 \text { - } 82\end{array}$} \\
\hline Discipline & \multicolumn{3}{|l|}{ Internet Of Things } \\
\hline Conference & \multicolumn{3}{|c|}{$\begin{array}{l}\text { A virtual international conference on redefining and transforming the role of higher } \\
\text { education in sustainable development }\end{array}$} \\
\hline Conference & \multicolumn{3}{|c|}{ Start Date: September 30, 2021} \\
\hline Dates & \multicolumn{3}{|c|}{ End Date : September 30, 2021} \\
\hline Institute Name & \multicolumn{3}{|c|}{$\begin{array}{l}\text { JAIN (Deemed-to-be University) in association with Council for Industrial } \\
\text { Innovation and Research }\end{array}$} \\
\hline $\begin{array}{l}\text { Date Received } \\
\text { ID } \\
\text { Dol }\end{array}$ & $\begin{array}{l}\text { : November } 19,2021 \\
: 2021.02 .19 \\
: 10.46402 / 2021.02 .19\end{array}$ & $\begin{array}{l}\text { Publication Date } \\
\text { Paper Type } \\
\text { Dol URL }\end{array}$ & $\begin{array}{l}\text { : December } 11,2021 \\
: \text { Conference Paper } \\
: \text { https://dx.doi.org/10.46402/2021.02.19 }\end{array}$ \\
\hline
\end{tabular}

\section{ABSTRACT}

Waste management is one of the world's most significant problems today, independent of rich or developing countries. One of the most frequent issues occurs when waste containers are fully overflowing before the following phase of cleaning starts. A smart waste disposal system is needed to avoid certain scenarios and to guarantee public cleanliness and hygiene. The ultrasonic sensor can detect/validate the filling level and the specific stature of the garbage in the trashcan. Programming is done in the Arduino UNO to get a cleaning message till a certain degree of filling is recognized. The study proposed an intelligent warning system to improve waste and cleanliness by delivering an instantaneous dustbin cleaning alarm signal to the municipal web server. The whole operation is regulated by a modules combined with RFID as well as IOT. The dustbin is in actual time and notifies the local authorities during collection, which is tracked and monitored with the use of this gadget.

KEYWORDS: Arduino Uno, COPD, Radio Frequency, Radio Frequency Identification, SGS. 


\section{INTRODUCTION}

Garbage management refers to appropriate waste treatment in a sustainable and costeffective manner. In accordance with the laws on waste management, this involves the purchase, treatment, transport and recycling of trash. Waste may be liquid solid, or gas, as well as individually form has a distinct dumping as well as management system. Garbage treatment covers all types of waste, including household, agricultural and environmental waste. Waste treatment. Proper sanitation, efficient and effective disposal of the remains generated is necessary. Due to inconsistent removal of trash occupancy, space for waste disposal has also been determined to be excessively busy.

In direction to deal with this problems, it is essential to have an e-monitoring solution including an embedded system and web apps using RFID and IOT technology. This structure enables the garbage level to be monitored at regular intervals in order to avoid inadvertent bin overflowing. Furthermore, it may also hint the capacity to clean up in case of spills. The ultrasonic sensor can detect/validate the filling level and the specific stature of the garbage in the trashcan. The Arduino UNO programming is carried out to get a cleaning message to identify a certain degree of fullness ${ }^{[1]}$.

The ultimate recycling innovation offers a new channels for updating the management of the waste infrastructure in a higher condition, especially with the RFID and sensor technologies. In current modern age, the increasing use of RFID innovation in many areas of application, such as public transit, logistics, inventory and security, can be observed to an extraordinary degree. In essence, RFID scans multimedia information with the assistance guiding near the papers from one or many objects. This enables you to programme ID, test, monitor property. It is stated that RFID may replace bar codes and may be introduced to a broad stock by manufacturers and merchants. These experiments helped bring forth a spectacular gadget, the Internet of Things ${ }^{[2]}$.

Connectivity via the Internet has now moved from users to device connections. Years before, the concepts of IoT were proposed, but they are only at the early stage of commercial application. In the home automation sector and transport industries, loT is experiencing significant growth. However, there haven't been many publications published in this area of research. This equipment needs active high speed internet connection and a large part of the operation takes place over the internet. Technology may be described simply as a connection between human computers. All devices used by people may be controlled and tracked in daily life via the IoT. IoT sensors take care of the rest of the operation. Sensors are installed throughout the facility, as well as these detectors turn raw physical data into digital signal, which are then sent to the control center ${ }^{[3]}$. 
Inappropriate waste disposal is, to some extent, responsible for air pollution and soil defilement, with complicated consequences on human health and expanding ecological deterioration. A study commissioned by a major Indian magazine showed clearly that the main reason for the hazardous air pollution in the town of Guwahati is the buildup of waste. The objective of this contamination in this township was to address the serious health issues discovered by the individuals who work in it, such as Chronic Obstructive Pulmonary Disease (COPD, for example) and Asthma.

The only purpose of mosquito and housefly reproduction is to prevent accumulated waste from being eliminated, which is the root cause of a large number of fatal illnesses including flu, dengue, and chikun guniya etc. There will never be a place to live in a city with bad sanitation and dangerous circumstances. Currently, around 235 million people are plagued by lung illness as a result of breathing foul-smelling air inside. Nearly 90 percentages of COPD sufferer are from little as well as middle-income countries. According to a health's publication, around $3,000,000$ peoples died of COPD in the year 2005.

The secret to a "intelligent city" is the ultimate time required for a rising nation. Dangerous pollution as well as its effects on human healths, global warming, ozone exhaustion, and other ecological challenges may pose a danger. The municipal solid residues may be mostly due to environmental contamination (MSL). Proper maintenance is necessary in order to efficiently and efficiently remove the produced solid municipal remains. It is believed that the trash area is frequently too much filled because of the irregular elimination of waste in the dustbins. This exhibition offers an E-monitoring systems that provides a web-based system and software equivalent to RFID and loT technologies ${ }^{[4]}$.

Monitoring the status of trash collection may be efficiently tracked using the expected system. This design specifies a mechanism for monitoring the garbage level at regular intervals in order to prevent the bin from overflowing. It also has the capacity to notify the appropriate authorities in the case of an overflow. The ultrasonic sensor can monitor / detect the dustbin's filling level as well as its beginning level. When a particular quantity of fullness is observed, the Arduino UNO is programmed to send out a cleaning message.

With the population growth of emerging countries apparently on the rise, the appropriate supervision of the Municipal Solids Leftover (MSL) is growing rapidly to construct and maintain an environmentally sound hazardous space. In the conventional method, a vehicle clears the discharged waste whenever a waste overflow is detected manually. But it is not checked sometimes. Regularly monitoring truck as well as recording the information that is relevant to the collection of time as well as area at a central places is extremely essential to guarantee that the work is done correctly ${ }^{[5]}$. 
This article demonstrates how RFID technologies may be utilized to develop an electronic monitoring systems to solve the issue in a traditional ways. An embedded systems with an RFID interface and an Arduino microcontroller, as well as a fully computerized web base, is the preferred E-monitoring systems. By using the suggested method, the local authorities could efficiently monitor the status of trash collection. There are two components to the e-monitoring system:

1. System embedded

2. Web-based system interface software

\section{Related Studies:}

The initiative aims to obtain dustbin information in real time. The management of garbage in real-time using intelligent dustbins to monitor the dustbin's fill level in order to determine whether or not the waste management system has been deployed using IOT. Providing administrations with the many types of managers in this area concerned. This book shows how the system for intelligent garbage management has been improved so that waste level is constantly measured in the trash container and, in particular, the area is warned by mean of SMS ${ }^{[6]}$.

The ultrasonic waste level calculation sensor, the GSM module that sends SMS, and the Arduino Uno that controls the framework's operation are all included in the recommended framework. The nutrient waste computation is meant to eliminate the loT-based intelligent waste system, according to the literature (SGS). Smart garbage receptacles powered by batteries communicate data over wireless networks in an SGS and collect and evaluate data for supply via a router and server. In addition, the SGS employs a number of IoT methods that take into consideration customer satisfaction and increase battery life through two types of SGB activity: stand-alone and cooperation-based activities.

\section{Methodology:}

Next to the garbage lid is a cylindrical portion that has been postponed. In vertical interiors, the piston is free to travel up and down and to a predetermined degree. A plate in the chamber compresses the rubbish. The shape of this plates is determined by the conditions of the litter bins. By use of a side aperture in the compacting pad, the turn of the leaf may be hung upside down. The height of the turn of the leaf is smaller than the highest level. The plate may thus reach down to a certain degree in order to push the button and drop the basket in the trash. The first part of the trashcan is placed at a point that is relatively smaller than the limit mark ${ }^{[7]}$.

The dustbin entrance is surrounded by an upper connected to the lateral surfaces of the inside. This blocks the intake top, when the compressor reaches the highest pressure 
level, so that no trashes from outside may be released into the dustbin. Prudent care is used to prevent the garbage from degrading or destroying the functioning of the leaf switch unless the conditions are severe. The trashcans are composed of materials that may have anti-corrosive properties.

The microcontroller (Arduino Uno) is a board that uses the ATmega3288 microprocessor. It has 14 computerized input as well as output pins, six analogue inputs, a sixteen $\mathrm{MHz}$ ceramic resonators, a power connections, an ICSP header, as well as a catch resets. Their solutions incorporate components that assist the microcontroller in whichever way it is needed. You may use it by linking it to a computers through USB or by giving supply it with an $A C$ to $D C$ converters or a power storage.

The Arduino Uno is available in two different variants. The field's variations are shown. The FTDI doesn't use the USB-to-sequence driver chips, hence the UNO is an exception to all of its previous sheets. "Uno" is an Italian word that meaning "one" and was chosen to honor the approaching relief of Arduino 1.0.0. In the future adventure, the Uno as well as the type 1.0 are Arduino references modifications. The Uno is the newest USB-Arduino board and the Arduino phase's standard model.

\section{Identifications of Radio Frequency:}

RFID is a method of identifying radio frequencies (RFID). In addition, RF signifies "radio frequency" to summarize things, and IDs due to "identifier" which allow access, recognition, storage, reinventing, and disclosure of anything, such as a library book, via the radio wave guide.

RFID is a phrase that mentions to non-contact technology that uses radio wave to classify peoples or things in an instant. Although there are a few other ID methods, the most basic is to register an unrecognizable number on a radio wire-controlled microchip that a person or thing understands. The "RFID tag" is a radio wire and microchip combination that works in tandem with the "RFID reader."

A reader and one or more tags make up an RFID system. With the aids of the Reader's antennas, radio frequency (RF) life is conveyed. The tag will then alter the reader's electromagnetic waves, and the tag will be totally transformed to provide the reader's integrated information. The modulated waves of digital information are presented to the reader $^{[8]}$.

\section{Working:}

The microchip determines the unique ID and houses the logic circuit that allows the tag to function correctly. It contains a built-in EPROM that keeps track of the UID. The antenna takes the RE signal as well as energy from the RFID reader and transfers it to 
the HT chip. The RFID reader receives these signals, validates them, and then sends them back to the chip.

The coil functioning as a tag antenna provides radio signals to the RFID reader. The signal is received by the coil, which then sends it to the chip as an AC current. This AC current provides both power and data to the chip. It interacts with a non-volatile chip memory, which carries a unique ID and other information, to send the needed signals to the antennas, which is subsequently transferred to the RFID readers.

The reader may also communicate the tag ID to an external device through sequential contact. A wide choice of reader modules is now available. The most common and simpleto-use reader is the EM-18. This module reads the RFID tag and sends the label ID to the external device via sequential contact. Currently, a wide selection of reader modules are available. The most popular and simple-to-use reader is the EM-18. The RFID tag is read by this module, which then provides the label ID to the Arduino microcontroller ${ }^{[9]}$.

Readers as well as RFID tags must be tuned to the similar frequency in order to communicate. The RFID system employs a number of frequencies, the most common of which are low, high, and ultra-high. In certain applications, a microwave frequency of 2.46 $\mathrm{GHz}$ is employed, with a low frequency of approximately $125 \mathrm{KHz}$, a high frequency of around $13.57 \mathrm{MHz}$, and an additional high frequency of 860 to $960 \mathrm{MHz}$. Because radio waves behave differently at various frequencies, selecting the appropriate frequency for a given application is crucial. The EM-18 RFID reader module creates and transmits 125 $\mathrm{KHz}$ frequencies RF carrier signals through its spools. When a passive $125 \mathrm{KHz}$ Rfk tag is attached, this field becomes active. In the manufacture of RFID tags, the EM4102 CMOS IC is often utilized ${ }^{[10]}$.

\section{DISCUSSION}

Waste management in the current century is becoming a serious problem. The main causes for increased trash production include an increase in population, lack of concern for the environment, poor resource management, etc. The problem continues to dominate, leading to different issues regardless of the established or developing country. We have four sub-sets of important concentrations in waste management. These include waste collection, monitoring, transformation and active processing for processing facilities. In every subset of a waste management concept, few difficulties emerge and they can be solved if processors are to be delivered and efficient processing in comparison with the other two subsets is carried out fairly fast. The problem in waste collection and monitoring is very complex since the waste is widely dispersed, produced extensively and mostly uncontrolled. Bins are the well-known and often used waste collecting techniques. These bins become the main problem and challenge to waste 
management owing to a lack of adequate monitoring and identification of their condition. Mull containers located at numerous locations are overflowing as the bin's threshold filling limit surpasses. This causes the waste to flow over to the area around the container and leads to unhealthy, filthy conditions. We should seek an effective waste collecting and bin drive system to prevent these problems, and to preserve our environment via clean and green methods. One potential method of addressing this is to constantly detect the waste bin filling state and to start an appropriate collection procedure before it is full. The usage of the Internet of things appears a preferable choice in delivering such intelligent solutions.

The Internet of Things (IOT) is the advanced technology emerging in a variety of applications. It combines information, communication and networking technologies via a single platform, i.e. the internet. In loT, everything is nothing but sensors which are linked to the service environment through a network integrated with the data transmission and storage system. IoT enables us to monitor any item situated remotely without any personal attention or visit. Here, the idea of IoT is also used to waste management in order to create a trash bins monitoring system. The purpose of the paper is to create the 'Smart Garbage Bin' (SGB) that allows us to monitor the quantities of trash filled in by transmitting information about their filing status with the 'Internet of Things' (IoT). Another goal is to check the prototype function by examining the test cases provided ( 0 percent or Empty, 50 percent or Medium, 90 percent or Nearly Full, 100 percent or Full, and Threshold Crossed or Spill Over).

In this article, we have built an loT-enabled waste collection container. The prototype was called "Smart Garbage Bin" and its performance was tested in order to validate its operating capacity after development. Experimental investigations have shown that SGB has shown that the validations in test cases and status identification are carried out in accordance with the intended consideration. Future work will be on implementing the 'Smart Garbage Bins' on a broad scale, taking into account the actual potential situations. Consider also the possibilities and effective operation of collection and cleaning services according to the size and complexity of the area of research. According to the current article, the usage of intelligent facilities will assist development in the relevant sector of the intelligent cities. The created intelligent trash bin facility may be utilized in the smart city, particularly in the newly-planned Indian smart cities for efficient waste management. However, deploying SGBs on a wider scale may provide a variety of difficulties, which are yet such as delays in bin cleaning if many containers are filled or dispersed.

\section{CONCLUSION}

A built-in intelligent warning system is utilized to maintain and properly monitor waste. The approach guarantees that irregular cleaning of market dustbins is monitored by 
providing periodic alerts to the appropriate authorities. It boosts the cleaning crew's performance and enhances the status of regular cleaning. As a result, this technology is a wonderful solution for environmental maintenance. In municipal waste management and pollution monitoring systems, it also helps to reduce the need for high human response levels. A built-in intelligent warning system is meant to keep track of and correctly manage waste. By delivering frequent warnings to the person in charge, this system protects the dustbins from being cleaned improperly. It also progresses the systems by monitoring the cleaning status in real time as well as evaluating the team's performance. This technology is thus an excellent option for environmental upkeep. In addition, it also helps reduce the requirement for significant human involvement in municipal waste management and pollution monitoring. 


\section{REFERENCES}

[1] $Y$. Wang and $X$. Zhang, "Autonomous garbage detection for intelligent urban management," 2018, doi: 10.1051/matecconf/201823201056.

[2] “Intelligent Garbage Classifier," Int. J. Interact. Multimed. Artif. Intell., 2008.

[3] A. Sathish, M. Prakash, S. A. K. Jainulabudeen, and R. Sathishkumar, "Intellectual trash management using Internet of Things," 2018, doi: 10.1109/ICCPEIC.2017.8290338.

[4] S. S. Nikam and P. B. Mane, "Swarm intelligent WSN for smart city," 2017, doi: 10.1007/978-981-10-1678-3_66.

[5] V. K. Ojha, P. Dutta, H. Saha, and S. Ghosh, "Linear regression based statistical approach for detecting proportion of component gases in manhole gas mixture," 2012, doi: 10.1109/ISPTS.2012.6260865.

[6] A. U. Kulkarni and G. N. Jayalaxmi, "IoT Solution for Live Wire Tampering," 2018, doi: 10.1109/PUNECON.2018.8745414.

[7] Y. Fan et al., "Study of possible usage of Finnish building technology in Chinese building development," VTT Publ., 1999.

[8] H. Rimminen et al., "MIT OpenCourseWare http://ocw.mit.edu Haus, Hermann A., and James R. Melcher.," Lect. Notes Comput. Sci. (including Subser. Lect. Notes Artif. Intell. Lect. Notes Bioinformatics), 2012.

[9] Ö. Göçer et al., "A Ship in a Box.," Appl. Mech. Mater., 2014.

[10] M. Lin and S. Chen, "Efficient and intelligent garbage collection policy for NAND flash-based consumer electronics," IEEE Trans. Consum. Electron., 2013, doi: 10.1109/TCE.2013.6626235. 